Revue d'histoire de l'Amérique française

REVUE D.HISTOIRE DE L'AMÉRIQUE FRANÇAISE

\title{
Un témoin de la dispersion acadienne : l'abbé LeGuerne
}

\section{René Baudry}

Volume 7, numéro 1, juin 1953

URI : https://id.erudit.org/iderudit/301576ar

DOI : https://doi.org/10.7202/301576ar

Aller au sommaire du numéro

Éditeur(s)

Institut d'histoire de l'Amérique française

ISSN

0035-2357 (imprimé)

1492-1383 (numérique)

Découvrir la revue

Citer cet article

Baudry, R. (1953). Un témoin de la dispersion acadienne : l’abbé LeGuerne. Revue d'histoire de l'Amérique française, 7(1), 32-44.

https://doi.org/10.7202/301576ar d'utilisation que vous pouvez consulter en ligne.

https://apropos.erudit.org/fr/usagers/politique-dutilisation/ 


\section{UN TÉMOIN DE LA DISPERSION ACADIENNE: L'ABBÉ LEGUERNE}

Les manuels d'histoire représentent quelquefois l'expulsion des Acadiens comme un événement bien déterminé, arrivé à GrandPré et dans les villages environnants, du 10 août au 30 octobre 1755. Ramenée à ces limites, la déportation aurait déjà été un acte inhumain, digne précédent des atrocités allemandes et russes de la dernière guerre. Mais cette barbarie ne fut pas si douce (peut-on dire ?) ni si facile. On n'expulse pas un peuple comme on chasse un mauvais locataire. Lawrence, dans une lettre aux Lords du Commerce, le 18 octobre 1755, se vantait qu'à la fin du mois suivant il ne resterait pas un seul Français dans ces régions. Or, à la fin de novembre, sur une dizaine de mille habitants qui peuplaient l'Acadie continentale, Lawrence et ses auxiliaires en avaient bien déporté quatre ou cinq milliers, mais il en restait pour le moins autant, qui se tenaient sur leurs gardes, au Cap Sable, à la Rivière Saint-Jean, dans la région de Memramcouk et Petcoudiac, ou réfugiés sur les côtes du Gaspé et à l'Ile Saint-Jean. Il fallut sept ans d'incursions, d'incendies et de chasses à l'homme avant de libérer à peu près le territoire.

Cette sombre période qui va de novembre 1755 à 1763 est peu connue. Si les documents abondent concernant la première phase de la Déportation, ils deviennent beaucoup plus rares sur les douloureuses péripéties qui la prolongèrent. Car il se trouva peu d'annalistes pour les relater. Dans toute l'étendue actuelle du NouveauBrunswick et de la Nouvelle-Écosse, il ne restait que deux officiers français, Boishébert et Niverville, avec une poignée de soldats, et quatre missionnaires, les Pères Germain et Labrosse, jésuites, et les 
abbés Manach et LeGuerne. Leurs témoignages, avec ceux des officiers anglais et la tradition orale, forment les uniques sources de renseignement sur ces noires années. L'un des témoins directs des événements, peut-être le plus complet et le plus impartial, est l'abbé LeGuerne. L'étude de sa vie et de ses lettres nous donne une bonne idée de l'époque 1753-1757 dans la partie la plus contestée de l'Acadie, l'isthme de Chignectou.

\section{Débuts missionnaires}

La première mention que nous trouvons au sujet de l'abbé LeGuerne, dans les lettres de l'abbé de l'Isle-Dieu', date de 1750. Il étudie alors au Séminaire du Saint-Esprit et nous apparaît comme un jeune Breton pauvre et rude. L'abbé de l'Isle-Dieu, cherchant de bonnes recrues pour l'évêque de Québec, dont il était Vicaire général, l'avait remarqué et s'était constitué son protecteur. Il l'envoya au Séminaire des Missions étrangères à Paris, en juillet 1749, et paya quelque temps lui-même sa pension dans cette maison. A plusieurs occasions, il recommande chaudement son protégé à Mgr de Pontbriand: "C'est un excellent sujet, dit-il, l'un des meilleurs de ceux qui se destinaient aux missions de Chine. Il n'a pas beaucoup de monde mais plus d'esprit qu'il n'est façonné."

Le jeune clerc, encore simple tonsuré, vint au Canada en mai 1750, y termina sa théologie et fut ordonné prêtre en septembre 1751. Il demeura environ deux ans à Québec et, comme l'abbé Leloutre, surchargé par ses multiples fonctions de Grand-vicaire pour l'Acadie, de missionnaire des sauvages et d'homme d'état, réclamait des renforts, Mgr de Pontbriand, au printemps de 1753, lui dépêcha le jeune abbé LeGuerne pour lui prêter main-forte. Celui-ci desservit d'abord un poste près de Beauséjour, Tintamar (Sackville) sur la rivière du même nom. Un an plus tard il prit charge des trois missions voisines, Chipoudy, Petcoudiac et Memramcouk, laissées vacantes par le départ de M. DuGuet.

En 1755, au moment de la Dispersion, il dessert donc depuis deux ans toute cette région de l'isthme acadien, que sillonnent les

1. Lettres de l'abbé de l'Isle-Dieu, dans RAPQ., (1935-36): 295-5, 308, 383; $(1936-37): 356,402$. 
quatre rivières, affluant à la Baie de Chignectou. Et il y trouve de quoi s'occuper. Son territoire comprend quarante lieues d'étendue; il a sous sa garde plus de 300 familles, soit près de 2,000 âmes, réparties en quatre principaux villages. Sans cesse il lui faut voyager par les rivières et les portages, et il emploie plusieurs mois de l'année à pèleriner d'un poste à l'autre. Il ne peut suffire à sa tâche que grâce aux desservants du fort Beauséjour qui viennent l'aider à tour de rôle.

Chaque jour la population de ses missions augmente par l'afflux des immigrants venant de l'Acadie anglaise. Le recensement de 1754 y indique, pour cette seule année, 35 nouvelles familles et une douzaine d'immigrants isolés. Il a fallu bâtir à Chipoudy une église neuve dont l'abbé LeGuerne est très fier. Dans les marais de Tintamar, et aussi à Memramcouk et Petcoudiac on construit, comme sous le fort Beauséjour, des digues pour récupérer de grandes étendues de terres fertiles et $y$ installer les nouveaux colons. Toute cette région, malgré les menaces de guerre, connaî́t un rapide développement. L'abbé LeGuerne besogne donc avec ardeur. Le soin de son troupeau occupe tout son temps, et les affaires de Dieu ne lui laissent guère de loisir pour s'occuper des affaires du roi. Son abstention de la politique lui vaudra les compliments intéressés de Pichon et de Moncton.

Surviennent les attaques anglaises et la chute du Fort Beauséjour (15 juin 1755). L'abbé Leloutre s'enfuit à Québec; un autre missionnaire suit les troupes, et l'abbé LeGuerne se trouve tout à coup "le seul prêtre et le seul Français" au centre de l'Acadie occupée, et dans la région la plus exposée. Que faire? Il choisit de demeurer avec ses gens. "Caché et fugitif avec eux dans les bois, écrit-il, dans la crainte et la misère, j'ai partagé avec les Acadiens qui y sont restés le triste sort où ils sont réduits, les aidant tous de mes conseils et de tout ce qui dépend de mon ministère". Attitude émouvante du pasteur fidèle qui affronte le danger, plutôt que d'abandonner son troupeau.

Afin de tenir ses supérieurs laïques et religieux au courant de la situation et aussi de les intéresser au malheureux sort de ses ouailles, il adresse de longs mémoires au gouverneur Vaudreuil ainsi qu'à l'évêque de Québec, et il entretient une fréquente correspondance avec les autorités de l'Isle Royale. Trois de ces lettres entre autres, 
datées de 1755,1756 et 1757 , sont très développées et forment une chronique complète des événements.

La première, adressée à l'abbé de l'Isle-Dieu, et probablement aussi à Vaudreuil, semble perdue. L'abbé Casgrain, dans Une Seconde Acadie (p. 307), en donne un résumé probablement imaginaire et reconstitué d'après d'autres documents.

La deuxième, écrite au camp de Bélair, près de Cocagne, en mars 1756, a été retrouvée aux Archives des Colonies et reproduite plusieurs fois ${ }^{2}$.

La dernière, moins connue, ne nous a été conservée que par un brouillon retrouvé dans les papiers de l'abbé LeGuerne, aujourd'hui à la paroisse Notre-Dame de Québec, à laquelle il avait légué sa bibliothèque. Elle a été publiée en 1889 avec une introduction biographique par Mgr C.-O. Gagnon ${ }^{3}$.

En analysant attentivement ces lettres, et en les complétant par quelques détails d'autres récits contemporains, comme le journal de Boishébert et les lettres de Vaudreuil, on peut se faire une idée exacte des deux premières années du martyre acadien en ces régions. C'est un chapitre d'histoire vu en toute crudité, frémissant de vie, et en même temps chargé d'angoisse.

\section{L'embarquement}

Dans les semaines qui suivirent la prise du Fort Beauséjour, les commandants anglais se firent apporter toutes les armes des Acadiens, puis, à diverses reprises, essayèrent sans succès de leur faire prêter le serment d'allégeance sans condition. Déjà le plan de l'expulsion était arrêté.

Le 10 août, "sous prétexte de prendre des arrangements pour les terres", Moncton, qui commandait au Fort Beauséjour, rebap-

2. Le texte original est aux Archives des Colonies, série C11A, 87: 388. Il a été reproduit une première fois par l'abbé H.-R. Casgrain, dans les Documents inédits du Canada-français, 2: 148, puis par Pl. Gaudet dans RAC, 1905, 2: 409, et par Henri d'Arles, dans Acadie, 3: 458.

3. Lettre de M. l'ab. LeGuerne, missionnaire de l'Acadie..., publiee par Mr C.O. Gagnon, prêtre de l'Archevéché de Québec. (Québec, Coté, 1889). Les citations sans référence, au cours du présent article, proviennent de cette lettre et de la précédente. 
tisé Fort Cumberland, convoqua tous les habitants des environs et leur déclara brutalement que "leurs terres, leurs biens, et leurs effets étaient confisqués au profit de la couronne et eux-mêmes faits prisonniers; puis les portes du fort se fermèrent et au-delà de $400 \mathrm{hom}$ mes furent ainsi emprisonnés" (Journal de Winslow 4,11 aout).

Ces prisonniers venaient surtout des environs immédiats de Beauséjour. Presque tous les hommes de Weskok, Aulac et Baie Verte s'y étaient rendus et il en était venu de plus loin, des trois rivières de Chipoudy, Petcoudiac et Memramcouk. Afin de ne pas trop ameuter la population, on fit d'abord croire aux prisonniers qu'ils allaient être transportés à l'Ile St-Jean. Lawrence, afin de diminuer la dépense, avait recommandé qu'on laissât lesfamilles des prisonniers leur apporter des vivres. Il était sûr ainsi de les avoir sous la main quand viendrait le moment de les saisir.

$\mathrm{Au}$ début, l'abbé LeGuerne hésita sur le parti à prendre et, suivant l'avis que Vergor et Leloutre avaient donné en partant, il conseilla aux Acadiens la soumission et l'obéissance. Il vécut aínsi deux mois sous la nouvelle domination et rendit même à Moncton quelques visites de courtoisie. Celui-ci, croyant le gagner à la cause anglaise, lui fit des promesses et lui offrít même des présents. Mais quand LeGuerne vit cet officier emprisonner les habitants, il comprit sa ruse et conseilla fortement aux Acadiens la résistance et la fuite.

On commença d'embarquer les prisonniers vers le 10 septembre. Chaque semaine, de petits groupes d'une cinquantaine à la fois montaient sur les vaisseaux. Ici, comme à Grand-Pré, l'embarquement donna lieu à des scènes pénibles. Comme le temps du départ approchait "de malheureuses femmes... se jetèrent aveuglément et comme par désespoir dans les vaisseaux anglais". Il s'en embarqua ainsi volontairement, entre 150 et 200.

L'Abbé LeGuerne entreprit alors de protéger ce qui restait de son troupeau. Il dut d'abord se protéger lui-même et se garder de tomber entre les mains de l'ennemi. Il prit donc ses hardes à Tintamar et se retira vers Memramcouk. A deux reprises Moncton

4. Journal of Col. John Winslow. Original aux Archives de la Massachusetts Historical Society, Boston. Reproduit dans Collections of the Nova Scotia Historical Society, Halifax, 3 (1883): 71-196; 4 (1885): 113-246. Extraits dans RAC., 1905, 2: 65-95. 
lui envoya des lettres insidieuses pour l'inviter à lui renouveler visite. LeGuerne lui rappela poliment l'arrestation des autres missionnaires et ses raisons de se méfier. Il conseilla aux femmes dont les maris avaient été emprisonnés, de ne pass se rendre aux Anglais, et à tous ses gens de se cacher, en attendant les ordres du Gouverneur.

\section{Escarmouches et incursions}

Vers la fin du mois d'aout, Boishébert ${ }^{5}$ apprenant le sort fait aux habitants de Beauséjour et des environs, vint de la rivière SaintJean avec une petite poignée de soldats et une centaine de sauvages malécites, formant en tout un détachement de 125 hommes. Le 27 août, le Col Frye avec 300 hommes venait ravager les établissements de Chipoudy et de Petcoudiac. Boishébert en atteignit un groupe au moment où ils brûlaient l'église, en tua une quarantaine sur place, en blessa un bon nombre, et d'autres périrent noyés en regagnant à la hâte leurs embarcations. "Ce coup, dit LeGuerne, fit plus trembler les Anglais que tous les canons de Beauséjour". A vrai dire les canons de Beauséjour n'avaient pas été bien terribles. Et les Acadiens purent récolter une partie de leur moisson.

Le 30 septembre, 86 prisonniers s'évadèrent du Fort Cumberland. Ils avaient creusé un étroit passage d'une trentaine de pieds sous le rempart, et s'enfuirent par là, à la faveur d'une nuit de tempête. Cette fuite mortifia grandement Moncton, car, malgré tous ses efforts, le nombre des prisonniers n'augmentait pas vite. Les partis qu'il envoyait en chasse ici et là ne remportaient que de minces succès. Au cours de l'automne, ils vinrent plusieurs fois en incursion dans les missions de LeGuerne. Mais le Gouverneur Vaudreuil avait donné l'ordre aux habitants de se retirer dans les bois, à une demilieue de leurs maisons; et les soldiats de Moncton ne purent récolter que quelques prisonniers, le plus souvent des femmes ou des jeunes gens. Pour se reprendre, ils se livrèrent avec ardeur au plaisir de

5. Sur Boishébert, voir son Journal (1755), Colontes C 110, 8: 222, reproduit dans RAC., 1905, 2: 236; les lettres de Prévost Drucourt et Vaudreuil (infra, notes 7 et 8); le Mémoire dans l'affaire du Canada pour le Sieur de Boishebert, par le Sr Clos, Paris 1763. Une traduction de ce Mémoire a été publiée par le Dr J.C. Webster, sous le titre de Boishebert Memorial, St. John, N.B., New Brunswick Museum, 1942. 
brûler les maisons et de razzier les bestiaux. Les journaux de Willard, Thomas et Scott nous ont conservé le récit détaillé de ces prouesses ${ }^{6}$ :

Le 15 nov. 1755, l'église de Tintamar et 97 maisons sont incendiées;

le 17 nov. expédition de nuit à Memramcouk; 30 maisons brâlées, une femme emmenée prisonnière, 200 têtes de bétail, 20 chevaux... le 20 nov. à Weskok, 100 maisons brúlées... etc. etc.

\section{Cachés dans les bois}

Vaudreuil, qui ne pouvait envoyer aucun renfort, donna ordre à Boishébert de s'établir en camp volant pour protéger les Acadiens et harceler les troupes anglaises par tous les moyens. Boishébert établit donc son camp à Cocàgne, lieu de rencontre des sauvages, et, de là, avec ses bandes harcelait l'ennemi à chaque occasion. Il y demeura du début de novembre à la mi-février.

Cette petite guerre de surprises et d'embuscades prit vite un caractère de férocité. Si les Micmacs et les Malécites ne martyrisaient pas leurs prisonniers, ils avaient cependant le casse-tête facile et pratiquaient avec adresse l'art du scalp. Les Rangers et leurs commandants, eux aussi de mœurs assez rudes, voulurent rivaliser de cruauté avec les sauvages, et donnèrent des ordres sanglants. Un évadé de Beauséjour, Pierre Suret, relata qu'à une excursion vers Memramcouk,

"le commandant de ce party avait ordre de se saisir de tous les Acadiens dans cet endroit, de faire mourir incontinent tous ceux qui s'y trouveraient en état de porter les armes, de leur lever la chevelure... d'emmener tout le reste après avoir laissé au bout d'un piquet une lettre pour M. de Boishébert, à peu près de ce style: Vous avez commencé, nous continuons sur ce même ton jusqu'à ce que vous vous retiriez de ce canton avec vos sauvages. On dit chez vous aux sauvages qu'autant d'Anglais qu'ils tueront, ce sera autant d'échelons pour aller en Paradis. Nous ajoutons que c'en sera deux pour nos gens par autant d'Acadiens qu'ils détruiront."

6. a) Journal of Abijah Willard (1755), dans Collections of the New-Brunswick Historical Society, 13 (1930): 3-75.

b) Diary of John Thomas (1755), dans Collections of the Nova Scotia Historical Society, 1 (1878): 119-140.

c) The Report and Map of Major George Scott's expedition... (1758), dans Collections of the New Brunswick Historical Society, 13 (1930): 97-114. 
Les milkciens anglais s'exercèrent à l'art du scalp. Il est fait mention dans la lettre de LeGuerne de deux hommes tués et scalpés à Chipoudy en 1756 et l'on trouve quelques témoignages du même genre dans les journaux d'officiers anglais ${ }^{7}$.

Cette vie de perpétuelles alertes et d'inquiétudes n'était guère confortable pour les Acadiens. L'abbé LeGuerne qui partageait leur vie dans les bois, nous a laissé un tableau de leurs misères. Presque tous avaient de proches parents partis pour l'exil; la plupart avaient vu flamber leurs maisons et tous leurs biens. Ils s'étaient construit des chétives cabanes en forêt et vivaient à la façon des sauvages. Mais à la longue leur patience se lassait et ils oubliaient le danger. Chaque fois qu'était pris un prisonnier, les autres devaient changer de refuge, même au fort de l'hiver, afin de dérouter l'ennemi. Le missionnaire se plaint de "la triste conduite des ha'bitants qui ne gardent pas la retraite et retournent chaque jour à leurs anciennes maisons". Boishébert dût menacer d'imposer de lourdes amendes aux imprudents et de les envoyer à Québec.

Les Acadiens avaient aussi à souffrir de la faim. Au cours de l'hiver de 1755 à 1756 , avec leurs animaux et le peu de blé qu'ils avaient récolté, ils auraient pu subsister assez facilement. Mais les patrouilles anglaises leur enlevèrent un bon nombre de bestiaux et le manque de fourrage rendait les animaux si maigres qu'ils avaient de la peine à se traîner.

De plus il fallait prélever sur ces approvisionnements des vivres pour nourrir les quatre ou cinq cents sauvages que Boishébert retenait en ces parages pour les incursions. Et ces sąuvages coûtaient terriblement cher à nourrir. Vaudreuil avait bien envoyé des fonds, mais ils furent vite engloutis. La rareté et la mauvaise qualité des aliments provoquèrent une épidémie, dont plusieurs personnes moururent.

A travers ces bouleversements, les missionnaires continuaient de remplir leur ministère. L'abbé LeGuerne avait cru pouvoir demeurer à Memramcouk, mais il craignit bientôt pour sa sûreté et se retira vers Cocagne. Le Père Labrosse vint de la rivière SaintJean prendre charge des missions de Petcoudiac. Un mince registre paroissial de cette région, conservé à l'archevêché de Québec, laisse

7. Voir: Knox, An Historical Journal..., (Champlain Society) 1: 196-7, note 3. 
voir un peu dans quelles conditions les missionnaires vaquaient à leur office. $\mathrm{Au}$ cours des actes laconiques, on peut lire des détails comme ceux-ci, du Père Labrosse:

Le 24 mars 1746: Dans la maison de Toussaint Blanchard, où à cause de la guerre, je tenais chapelle...

Le 14 mars, 1756: Dans une chapelle que les habitants de Chipoudy refugiés au bois avaient bâti...

Le 21 mars, 1756: Dans une chapelle que les habitants de l'Isle de Pedkodiak refugiés au bois avaient bâti...

L'abbé LeGuerne, lui, n'a plus de cahier pour enregistrer ses actes; il note les baptêmes, sur des bouts de papier qu'il joindra plus tard au registre. Ces prêtres disent ainsi la messe, exhortent leurs fidèles, bénissent les tombes. Parfois les Acadiens ne peuvent même pas enterrer leurs morts en paix. "Si j'avais été à Memramcook, note l'abbé LeGuerne en marge de sa lettre, j'aurais été pris infailliblement à un enterrement où l'Anglais parut, tout à coup, en faisant une décharge de mousqueterie".

Le plus étonnant, c'est qu'en ces temps troublés, les missionnaires aient encore à célébrer des mariages. Des jeunes gens, malgré l'incertitude du lendemain, profitent d'une accalmie pour lier leur sort, et malgré tout, continuent d'espérer dans l'avenir et dans la Providence.

Il était évident cependant qu'abandonnés pratiquement sans défense par les gouverneurs impuissants de Québec et de Louisbourg, et devant la poursuite acharnée des troupes anglaises, les Acadiens ne pouvaient tenir longtemps; il fallait évacuer leur propre pays et chercher refuge ailleurs.

\section{L'évacuation}

S'ils n'avaient pu réussir complètement à convaincre leurs gens de prudence, ce fut encore bien pis quand Boishébert et LeGuerne entreprirent de leur conseiller d'émigrer. L'un des traits du peuple acadien, ce fut toujours l'attachement à son sol, à ses collines, à ses rivières, où près de la grande forêt giboyeuse et des prés fertiles, il vivait des jours heureux. "A les entendre on est misérable partout ailleurs, on y mange de viande que le quart de son saoul. L'Acadie, 
disent-ils, jusqu'à ces dernières années, était un paradis sur terre". Leurs maisons sont-elles brûlées, ils se construisent des cabanes. Ces cabanes détruites, ils en construisent d'autres. Ils s'accrochent à leur pays avec une espèce de désespoir. Toutes sortes de rumeurs contribuent aussi à augmenter leur indécision. Ils espèrent que la France n'abandonnera pas cette colonie et songera bientôt à la reprendre. Les Anglais, eux-mêmes, c'est Scott qui parle, disent "que le roi de France n'a point de sentiment, s'il ne tire point de vengeance de ce qu'on lui a fait dans l'affaire de Beauséjour."

Cependant, l'imminence du danger et les conseils des missionnaires finissent par en convaincre un bon nombre. Le Col. de Boishébert, le P. Germain et l'abbé LeGuerne travaillent de concert à favoriser leur évacuation. Dès l'automne de 1755 , le premier soin de l'abbé LeGuerne avait été de porter secours à une centaine de femmes et d'enfants des environs du Fort Cumberland, qui demeuraient avec leurs enfants exposés à l'ennemi, sans pouvoir ellesmêmes se transporter au bois avec leurs effets indispensables. Il réunit les jeunes gens, les vieillards et quelques évadés, et organisa une espèce de caravane qui "par des pays horribles et marécaux" entreprit à pied ou en charrette une longue "route de 10 lieues, pour se rendre au bord de la mer, vis à vis l'Isle St-Jean". Le voyage prit un mois et l'abbé LeGuerne les accompagne, les encourageant et les aidant à transporter leurs pauvres bagages.

Dans cet automne, environ 500 Acadiens du bassin intérieur de la baie de Fundy passèrent ainsi aux rives du Golfe, en face de l'Isle St-Jean. Villejoint envoya des bateaux les chercher dans la baie de Shédiac et les accueillit sur l'Isle St-Jean ${ }^{8}$. Les environs de Beauséjour se trouvèrent ainsi à peu près entièrement vidés.

Restaient les rivières de Memramcouk et Petcoudiac où demeuraient encore environ 250 familles. Aux premiers mois de 1756, devançant le printemps, LeGuerne invite ses gens à profiter des glaces pour se rendre au bord de la mer et y attendre les vaisseaux. Lui-même s'y rend afin de leur chercher un abri. Une soixantaine de familles de Memramcouk répondent à son appel. Au mois d'août de la même année, une soixantaine d'autres familles qui jusque-là

8. Voir la correspondance de Prévost et Drucourt dans Colonies C11D, Ile Royale, volumes 35-37, passim. 
avaient pu demeurer cachées aux environs des Mines et de PortRoyal, réussissent à traverser la Baie de Fundy et à se rendre à Petcoudiac par la rivière Saint-Jean.

Mais où les diriger? M. de Villejoint à l'Isle St-Jean a plus de monde qu'il n'en peut nourrir. Par ailleurs le gouverneur, ignorant les intentions du roi au sujet de l'Acadie, ne veut pas prendre la responsabilité de les faire passer au Canada. C'est alors qu'obéissant à certaines pressions intéressées, il donne l'ordre d'établir provisoirement les Acadiens à Miramichi. Ce que les Acadiens y souffrirent dépassa tout ce qu'ils avaient enduré auparavant. L'abbé LeGuerne s'était opposé à cette solution: "On a donc placé, écrit-il, les Acadiens qui ne pouvaient plus subsister dans leurs quartiers dans un endroit de misère, je veux dire à Miramichi où ces pauvres gens sont morts l'hiver dernier en grande quantité de faim et de misère, et ceux qui ont échappé à la mort n'ont pas échappé à une horrible contagion et ont été réduits par la famine qui y règne, à manger du cuir de leurs souliers, de la charogne et quelques-uns même ont mangé jusqu'à des excréments d'animaux". Une lettre de Vaudreuil ${ }^{9}$ complète ce tragique tableau.

\section{Epilogue}

A l'été de 1757, l'abbé LeGuerne prit le chemin de Québec. Probablement était-il dégoûté de ce gâchis auquel il ne pouvait rien. Il semble aussi que sa santé avait gravement souffert. Il voulait de plus intervenir lui-même auprès du Gouverneur. Il avait d'ailleurs rempli la majeure partie de sa tâche, puisqu'il ne restait plus dans ses missions qu'un petit groupe de familles dans le haut de la rivière Petcoudiac, et qui semblaient bien décidées à ne point quitter ces régions.

Aussitôt rendu à Québec, LeGuerne écrit à Vaudreuil pour lui demander des secours en faveur des Acadiens. Il prit quelques mois

9. La correspondance de Vaudreuil avec la Cour donne le résumé des dépêches qu'il recevait fréquemment d'Acadie, soit de Boishébert et de ses lieutenants, soit des missionnaires. Voir Colonies C 11A, 100: 86 \& seq. Plusieurs de ses lettres figurent dans RAC, 1905, 2: 237 \& seq. et dans la Coll. de Ms. relatifs à la Nouvelle-France vol. 4, passim. 
de repos, puis il fut question de l'envoyer aux missions de Louisiane, mais l'abbé de l'Isle-Dieu conseilla plutôt de le garder à Québec au cas où la France recouvrerait l'Acadie. Il n'y devait pas revenir. Mgr de Pontbriand le nomma, en 1758, curé de St-François, île d'Orléans, où il retrouva un petit groupe de ses proscrits établis là l'année précédente.

Ce Breton têtu devait éprouver pour les tenaces Acadiens une secrète et forte affinité. On verra aussi Boishébert garder au moins quelques années des relations avec les Acadiens. Il recruta parmi eux, en 1758, un parti pour aller secourir Louisbourg et, lors de la bataille de Sainte-Foy, il commandait un détachement d'Acadiens et de Micmacs. Cet officier retourna en France après les capitulations, mais l'abbé LeGuerne choisit de demeurer en Canada. Il enseigna quelque temps la Rhétorique au Petit Séminaire de Québec et fournit une longue carrière comme curé de Saint-François, puisqu'il ne mourut qu'en 1789, âgé de 64 ans.

Mais la période la plus remplie de sa vie coïncida sans doute avec les cinq années passées en Acadie. Là il avait donné toute sa mesure, celle d'une intelligence perspicace et d'une grande âme. Après son départ plusieurs petits groupes d'Acadiens fugitifs se rendirent ou furent capturés; mais il en demeura toujours un certain nombre, à la Rivière Saint-Jean et sur les côtes du Golfe et de la Baie-des-Chaleurs.

Quand Lawrence mourut en 1760, son œuvre n'était pas achevée, puisqu'il restait encore dans son territoire au moins un millier d'Acadiens. Et s'il vivait aujourd'hui, il lui faudrait recommencer toute sa besogne, puisqu'ils ne sont plus seulement 1000 , ni 15,000 , mais 300,000 .

L'âme de la résistance acadienne résida sans doute dans les qualités d'énergie et d'endurance du peuple acadien lui-même; mais cette résistance se noyauta autour de quelques chefs, très peu nombreux: des militaires comme Boishébert et Niverville, des prêtres comme le P. Germain, le P. Labrosse, l'abbé Manach, l'abbé LeGuerne.

Un détail ne peut manquer de frapper quand on cherche à mesurer le rôle qu'ils jouèrent, c'est l'extrême jeunesse de quelques-uns d'entre eux, Boishébert avait 28 ans, en 1755; l'abbé LeGuerne, 30 ans. 
Aux jeunes Acadiens d'aujourd'hui, qui fréquentent nos collèges, aux Provinces Maritimes ou dans la Province de Québec, descendants des paroissiens têtus de l'abbé LeGuerne et des miliciens qui servirent sous Boishébert, peut-on donner meilleur exemple de courage et de valeur que celui des deux jeunes chefs qui commandèrent à leurs ancêtres?

René BAUDRY, c.s.c. Universite Saint-Joseph, N.B.

AVIS A RETENIR : Nous tenons à la disposition des amis de la Revue, un certain nombre de tirés à part de Vue rétrospective (les 4 premières pages de la présente livraison signées par le président). Nous en enverrons volontiers à ceux qui voudraient nous recruter de nouveaux abonnés. 